\title{
A proposal of the gauge theory description of the small Schwarzschild black hole in $\mathrm{AdS}_{5} \times \mathrm{S}^{5}$
}

\author{
Masanori Hanada ${ }^{a, b, c}$ and Jonathan Maltz ${ }^{d, a}$ \\ a Stanford Institute for Theoretical Physics, Department of Physics, Stanford University, \\ 382 Via Pueblo, Stanford, CA, 94305 U.S.A. \\ ${ }^{b}$ Yukawa Institute for Theoretical Physics, Kyoto University, \\ Kitashirakawa Oiwakecho, Sakyo-ku, Kyoto, 606-8502 Japan \\ ${ }^{c}$ The Hakubi Center for Advanced Research, Kyoto University, \\ Yoshida Ushinomiyacho, Sakyo-ku, Kyoto, 606-8501 Japan \\ ${ }^{d}$ Berkeley Center for Theoretical Physics, Department of Physics, \\ University of California at Berkeley, 366 LeConte Hall, Berkeley, CA, 94720 U.S.A. \\ E-mail: hanada@yukawa.kyoto-u.ac.jp, jdmaltz@berkeley.edu
}

AbStRACT: Based on $4 \mathrm{~d} \mathcal{N}=4 \mathrm{SYM}$ on $\mathbb{R}^{1} \times \mathrm{S}^{3}$, a gauge theory description of a small black hole in $\mathrm{AdS}_{5} \times \mathrm{S}^{5}$ is proposed. The change of the number of dynamical degrees of freedom associated with the emission of the scalar fields' eigenvalues plays a crucial role in this description. By analyzing the microcanonical ensemble, the Hagedorn behavior of long strings at low energy is obtained. Modulo an assumption based on the AdS/CFT duality for a large black hole, the energy of the small ten-dimensional Schwarzschild black hole $E \sim 1 /\left(G_{10, N} T^{7}\right)$ is derived. A heuristic gauge theory argument supporting this assumption is also given. The same argument applied to the ABJM theory correctly reproduces the relation for the eleven-dimensional Schwarzschild black hole. One of the consequences of our proposal is that the small and large black holes are very similar when seen from the gauge theory point of view.

KEYWORDS: Black Holes in String Theory, Gauge-gravity correspondence, M(atrix) Theories

ARXIV EPRINT: 1608.03276 


\section{Contents}

1 Introduction 1

2 Stringy interpretation of the field theory degrees of freedom 4

2.1 Emission of D-branes and black hole evaporation 5

3 Analysis of the microcanonical ensemble $\quad 6$

3.1 The large black hole (large energy and $\lambda \gg 1$ ) 6

3.2 Emission of eigenvalues 6

3.2.1 Gauge fixing 8

$3.310 \mathrm{~d}$ Schwarzschild at $1 / \lambda \ll N_{\mathrm{BH}} / N<1(\lambda \gg 1) \quad 10$

3.3.1 The phase transition from the big black hole to the small black hole 12

3.4 The Hagedorn growth at $N_{\mathrm{BH}} / N \lesssim 1 / \lambda(\lambda \gg 1) \quad 13$

3.5 The case of weak coupling $\lambda \ll 1 \quad 13$

$\begin{array}{lll}3.6 & \mathrm{SO}(6) \text { breaking } & 13\end{array}$

$\begin{array}{lll}3.7 & \text { Other topologies } & 14\end{array}$

4 Other cases $\quad \mathbf{1 5}$

4.1 ABJM theory and 11d black hole $\quad 15$

$\begin{array}{ll}4.2 \text { More generic theories } & 16\end{array}$

5 Discussions 16

\section{Introduction}

Gauge/gravity duality [1] is believed to be a key idea in resolving the black hole information paradox. Witten [2] conjectured that $4 \mathrm{~d} \mathcal{N}=4$ super Yang-Mills (SYM) theory compactified on a three-sphere $\mathrm{S}^{3}$, whose action is given by

$$
S=\frac{1}{g_{\mathrm{YM}}^{2}} \int d^{4} x \operatorname{Tr}\left(\frac{1}{4} F_{\mu \nu}^{2}+\frac{1}{2}\left(D_{\mu} X_{M}\right)^{2}+\frac{1}{4}\left[X_{M}, X_{M^{\prime}}\right]^{2}-\frac{1}{2} X_{M}^{2}+(\text { fermion })\right),
$$

can describe a black hole $(\mathrm{BH})$ in $\mathrm{AdS}_{5} \times \mathrm{S}^{5}$. Here the gauge group is $\mathrm{SU}(N)$ and $X_{M}$ $(M=1, \cdots, 6)$ are $N \times N$ Hermitian matrices. We consider 't Hooft large- $N$ limit, $g_{\mathrm{YM}}^{2} \propto N^{-1}$, and the radius of the $\mathrm{S}^{3}$ is set to 1 . If the conjecture is correct, then the dual gravity description suggests the following behavior in phase diagram of the microcanonical ensemble at strong coupling (see e.g. section 3.4.1 of [3]):

- When the energy $E$ is large enough, a large AdS-BH, which fills the $S^{5}$ direction, is formed. The energy scales as $E \sim \frac{R_{\mathrm{AdS}}^{11} T^{4}}{G_{10, \mathrm{~N}}}$ at high temperature $T$, where $G_{10, \mathrm{~N}}$ and $R_{\text {AdS }}$ are the ten-dimensional Newton constant and the AdS radius, respectively. Note that the large AdS-BH has a positive specific heat. 


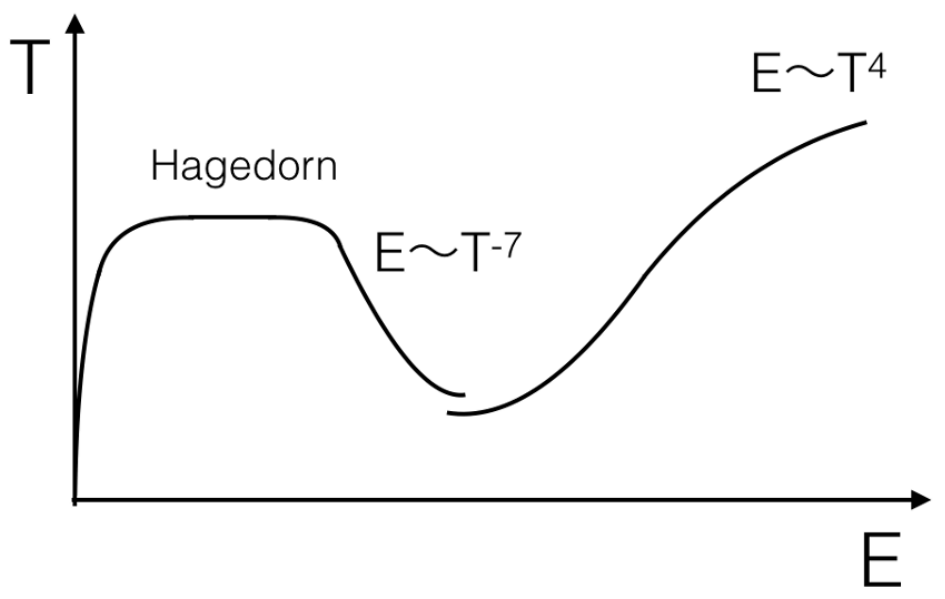

Figure 1. The microcanonical $E$-vs- $T$ phase diagram of $4 \mathrm{~d} S Y M$ on $\mathbb{R}^{1} \times \mathrm{S}^{3}$ at strong coupling $\left(\lambda=g_{\mathrm{YM}}^{2} N \gg 1\right)$, obtained by assuming the validity of the AdS/CFT duality.

- The large AdS-BH shrinks as the energy is decreased and the temperature goes down. When the Schwarzschild radius becomes of order $R_{\mathrm{AdS}}$, the $\mathrm{BH}$ localizes along the $\mathrm{S}^{5}$ and can be regarded as a ten-dimensional $\mathrm{BH}$. This transition is of first order [4], and the BH becomes hotter after the localization. ${ }^{1}$ When the Schwarzschild radius becomes much smaller than $R_{\mathrm{AdS}}$, the $\mathrm{BH}$ should behave like the ten-dimensional Schwarzschild $\mathrm{BH}$ in flat spacetime, $E \sim \frac{1}{G_{10, \mathrm{~N}} T^{7}}$. We will call this localized BH, the small $\mathrm{BH}$. Note that the small $\mathrm{BH}$ has a negative specific heat.

- As the small BH shrinks towards the string scale, the description of it as a bunch of long strings become better. The system shows the famous Hagedorn behavior, $E \propto S \propto L$, where $S$ is the entropy and $L$ is the length of the strings.

- Finally, when the energy is very small, the system is well described as a gas of short strings.

The relation between the energy and temperature is shown in figure 1 .

Although the gauge/gravity duality conjecture has not been proven, there is accumulating evidence that it is most likely correct. Hence the majority of string theorists believe that $4 \mathrm{~d}$ SYM has the same phase diagram. It is crucial however to understand this phase diagram directly from the gauge theory for several reasons. First of all, the duality has been poorly tested at finite temperature. As far as we know, the only quantitative tests currently understood $[5],{ }^{2}$ are for the duality [10] between the type IIA black zero-brane and D0-brane quantum mechanics [11-13], which is analogous to the large AdS-BH in 4d $\mathcal{N}=4$ SYM. (For the $\mathrm{AdS}_{3} / \mathrm{CFT}_{2}$ correspondence, the supergravity limit and the universal logarithmic correction have been studied from $\mathrm{CFT}_{2}$ side; see [14] and references therein.) Hence we need to test gauge/gravity duality for a small BH. Furthermore, if we use the duality to understand the quantum gravitational aspects of black holes, we have

\footnotetext{
${ }^{1}$ The authors would like to thank Jorge Santos for the clarification.

${ }^{2}$ Analytic approaches for deriving nontrivial temperature dependence have been discussed in [6-9].
} 
to solve gauge theory. If we assumed the validity of the dual gravity description and used the gravitational description to explain gravity, we would be just assuming the answer to answer the question.

Previously, the gauge theory description of the large AdS-BH, the Hagedorn parameter region, and string gas parameter region have been understood at least qualitatively (see e.g. [3]). In this paper, we propose a simple gauge theory description of the small BH. By assuming the validity of the AdS/CFT correspondence for the large black hole, we derive the relation between the energy and temperature of the small black hole, $E \sim 1 /\left(G_{10, \mathrm{~N}} T^{7}\right)$, at strong coupling. We also give a heuristic explanation supporting this assumption based only on gauge theory. In addition, we will show that the same picture correctly reproduces the Hagedorn behavior. In short,

- The large black hole is described by a bound state of all the eigenvalues of scalar fields $X_{M}$. All $N^{2}$ matrix entries are excited.

- Suppose some of the eigenvalues are emitted, after which and only $N_{\mathrm{BH}}<N$ eigenvalues form a bound state. Such matrices describe the small black hole. The black hole is smaller when $N_{\mathrm{BH}}$ is smaller. ${ }^{3}$

This paper is organized as follows. In section 2, we remind the readers how the microscopic, stringy degrees of freedom can be read off from the fields (matrices) in $4 \mathrm{~d}$ SYM. Two seemingly different, but actually equivalent, pictures — 'open strings + D-branes' and closed strings - are introduced, and the meaning of the emission of the D-branes (eigenvalues) from the $\mathrm{BH}[16,17]$ is explained. Section 3 is the main part of this paper. We propose a gauge theory description of the small black hole, and obtain the relationship between the energy, entropy and temperature expected from the conjectured gravity dual modulo a technical assumption explained at the end of the section. In section 4, we suggest that the same picture can hold for a rather generic class of theories holographically dual to superstring/M-theory. We study the ABJM theory as an example and derive the right energy-temperature relation of the 11d Schwarzschild black hole.

Note added. While this work has been in progress, we have learned that Leonard Susskind had essentially the same idea independently. He conjectured the small black hole is described by a small sub-matrix, and considered a possibility of making the 'box' (AdS space) smaller in order to remove the degrees of freedom which are not needed for describing the small BH. In terms of gauge theory, this means a truncation to $\mathrm{U}\left(N_{\mathrm{BH}}\right)$. Then he assumed the 'corresponding principle' which relates the large and small black holes. On the gauge theory side, mathematically, this is exactly what we have done in order to derive $E \sim 1 /\left(G_{10, \mathrm{~N}} T^{7}\right)$. We would like to thank him for stimulating discussions and collaboration toward the end of the project.

\footnotetext{
${ }^{3}$ The idea that the size of the matrix blocks changes with the energy has also been an important ingredient of a proposal for a description of the Schwarzschild black hole in the Matrix Model of M-theory [15].
} 


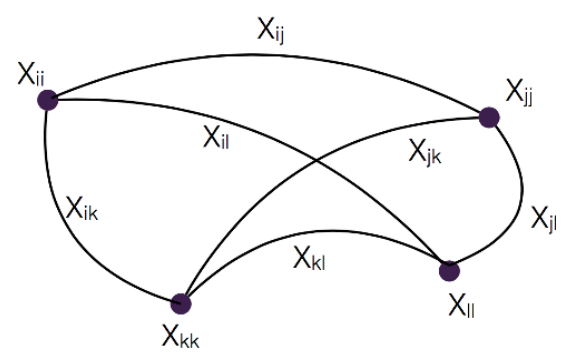

Figure 2. Interpretation of matrix components as sites and links of a nonlocal lattice.

\section{Stringy interpretation of the field theory degrees of freedom}

In this section, we explain how the stringy micro-states of a black hole are encoded into gauge theory. There are two seemingly different pictures, (1) the bound states of D-branes and strings [11] and (2) long, winding strings [18, 19]. Here we explain how they are related to each other.

Firstly let us consider the D0-brane quantum mechanics picture [11-13], the generalization to generic gauge theories including $4 \mathrm{~d} \mathcal{N}=4 \mathrm{SYM}$ is straightforward. We work in the Hamiltonian formulation [20]. The gauge field is set to zero, $A_{t}=0$, and the physical Hilbert space is obtained by acting traces of products of scalars $X_{M}(M=1,2, \cdots, 9)$ on the vacuum state. ${ }^{4}$ When we follow the usual D-brane effective theory point of view [11], the diagonal components $X_{M, i i}$ are regarded as the position of $i$-th D0-brane in $\mathbb{R}^{9}$, and the off-diagonal components $X_{M, i j}$ describe open strings connecting $i$-th and $j$-th D0-branes. Note that these strings are oriented. When $X_{M, i j}$ is large, a lot of strings are excited between $i$-th and $j$-th D0-branes. This is picture (1).

In order to go to picture (2), let us regard the D0-branes and open strings as sites and links of a non-local lattice (figure 2). Here we use the adjective "nonlocal" because all pairs of sites can be directly connected by links. Gauge-invariant states are made of closed loops. ${ }^{5}$ For example, if we consider $\operatorname{Tr}\left(X_{M_{1}} X_{M_{2}} X_{M_{3}} X_{M_{4}}\right)|V a c\rangle ; X_{M_{1}, i k} X_{M_{2}, k l} X_{M_{3}, l j} X_{M_{4}, j i}$ with different $i, j, k, l$ is a closed loop made of four links (figure 3), while $X_{M_{1}, i k} X_{M_{2}, k l} X_{M_{3}, l l}$ - $X_{M_{4}, l i}$ with different $i, k, l$ is a closed loop made of three links and one site (figure 4 ). Hence the black hole, which is a bound state of D-branes and open strings, is naturally regarded as a long, winding string in the lattice description. (More precisely, a few long strings.) The maximum possible number of string bits (open strings) is the maximum possible length of a single trace operator, which is of order $N^{2}$. This upper bound appears because single trace operators beyond this bound can be expressed by using shorter operators. Note that this is slightly different from the 'correspondence principle' $[18,22]$ in the usual sense, that the black hole is always described by strings in this picture $[18,19]$.

When the number of spatial dimension is nonzero, the gauge fields form other links; the picture should be clear if one imagines a lattice discretization of space-time. Then

\footnotetext{
${ }^{4}$ More precisely speaking, the adjoint fermions exist as well. The gauge-singlet condition follows from the Gauss-law constraint.

${ }^{5}$ This idea is not new. See e.g. [21].
} 


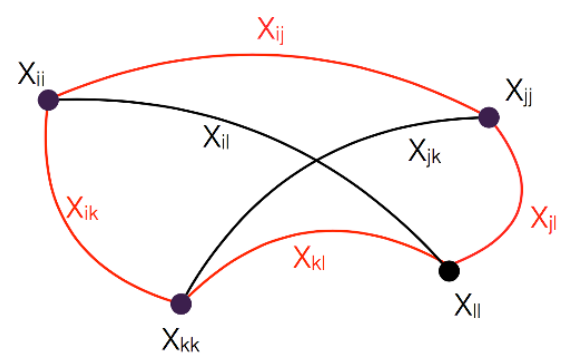

Figure 3. $X_{M_{1}, i k} X_{M_{2}, k l} X_{M_{3}, l j} X_{M_{4}, j i}$ in $\operatorname{Tr}\left(X_{M_{1}} X_{M_{2}} X_{M_{3}} X_{M_{4}}\right)|V a c\rangle$.

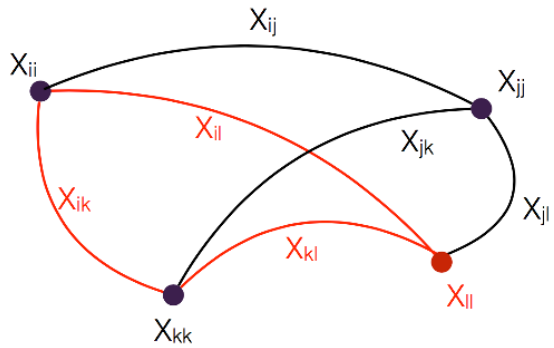

Figure 4. $X_{M_{1}, i k} X_{M_{2}, k l} X_{M_{3}, l l} X_{M_{4}, l i}$ in $\operatorname{Tr}\left(X_{M_{1}} X_{M_{2}} X_{M_{3}} X_{M_{4}}\right)|V a c\rangle$.

the link variables also become strings; generic long string states are expressed by Wilson loops (QCD strings) with scalar insertions. Here the black brane can be regarded as a condensation of long strings. For more details, see [19].

\subsection{Emission of D-branes and black hole evaporation}

The dynamics of D-branes play an important role in the matrix (gauge theory) description of the black holes [12]. Suppose the D-branes form well separated and localized bunches consisting of $N_{1}, N_{2}, \cdots$ D-branes. Then strings inside each bunch are short, light and can be very excited, while strings connecting different bunches are long, heavy and cannot be excited much. In terms of matrices, such a configuration is expressed by almost blockdiagonal matrices with block sizes $N_{1}, N_{2}, \cdots$. Each block is regarded as long strings with maximum length $N_{1}^{2}, N_{2}^{2}, \cdots$, respectively. Typically, the $i$-th block carries the energy and entropy of order $N_{i}^{2}$.

Next let us consider the emission of eigenvalues, following $[16,17] .{ }^{6}$ When one of the D-branes is emitted, open strings between the emitted D-brane and the others become heavy and decouple from the dynamics. Hence fully noncommutative $N \times N$ matrices turn to block diagonal forms,

$$
\left(\begin{array}{cc}
X_{\mathrm{BH}}^{M} & 0 \\
0 & x^{M}
\end{array}\right)
$$

\footnotetext{
${ }^{6}$ In the theories with flat directions, the emission is inevitable. In $4 \mathrm{~d} \mathcal{N}=4 \mathrm{SYM}$ on $\mathbb{R}^{1} \times \mathrm{S}^{3}$, though there are no flat directions, emission still plays an important role, as we will see shortly.
} 
where $X_{\mathrm{BH}}^{M}$ are fully noncommutative $(N-1) \times(N-1)$ matrices and $x^{M}$ describe the position of emitted D-brane. ${ }^{7}$ This is the Higgs mechanism. The number of light physical degrees of freedom decrease from $\sim N^{2}$ to $\sim(N-1)^{2}+1$, while the energy is conserved during emission. Hence the energy per degree of freedom $(\simeq$ temperature $)$ increases. As the emission continues more eigenvalues can be emitted and the black hole becomes hotter and hotter. The negative specific heat of the black hole follows from this simple fact. For details, see $[16,17]$.

In the next section, we show that the emission of D-branes can explain the properties of the small black hole in $\mathrm{AdS}_{5} \times \mathrm{S}^{5}$ described by $4 \mathrm{~d} \mathrm{SYM}$ on $\mathbb{R}^{1} \times \mathrm{S}^{3}$.

\section{Analysis of the microcanonical ensemble}

\subsection{The large black hole (large energy and $\lambda \gg 1$ )}

The large $\mathrm{AdS}_{5}$ black hole is obtained by rolling up a black three-brane with charge $N$. The black brane is a bound state of all $N$ eigenvalues (D3-branes); as is the large black hole. See figure 5. As we declared in the introduction, we assume the AdS/CFT duality between SYM and the large BH is correct. In principle (and within a ten-year span, probably in practice), the duality can be tested by Monte Carlo simulation. For recent numerical studies, see e.g. [23, 24].

When the black hole on the gravity side fills the $\mathrm{S}^{5}$ completely, the $\mathrm{AdS}_{5} \mathrm{BH}$ should be used in the dual gravity calculation. It has the minimum temperature $T_{\min }$; see figure 6 . Let us call the energy at $T=T_{\min }$ to be $E_{\min }$, and the solutions at $E>E_{\min }$ and $E<E_{\min }$ to be 'large' and 'small' $\mathrm{AdS}_{5}$ black holes, respectively. (Hence $E_{\min }$ is the minimum energy of the 'large' BH.) To avoid confusion we will use small BH to refer to a 10d Schwarzschild black hole and small $\mathrm{AdS}_{5} \mathrm{BH}$ to refer to a small AdS black hole which still fills the $\mathrm{S}^{5}$.

The small $\mathrm{AdS}_{5} \mathrm{BH}$ solution at $E<E_{\text {min }}$ does not have a counterpart in $4 \mathrm{~d} \mathrm{SYM}$; it is unstable with respect to the Gregory-Laflamme instability [25] along the $\mathrm{S}^{5}$, and hence the $10 \mathrm{~d} \mathrm{BH}$ becomes the appropriate description. From the point of view of gauge theory, it can be understood as follows. In order for a bound state of eigenvalues (non-commutative block) to be formed, (almost) all of the off-diagonal elements must be excited, which costs a lot of energy. Hence the $\mathrm{AdS}_{5} \mathrm{BH}$ black hole can exist only when the energy is large enough.

Strictly speaking, the 'minimum energy' $E_{\min }$ corresponding to the instability might be slightly different from the energy at $T_{\min }$; they should be of the same order but it is hard to determine the order one factor. A dual gravity analysis such as $[4,26]$ may provide us with a concrete number. In the following we will not consider order one coefficients which are sensitive to this ambiguity.

\subsection{Emission of eigenvalues}

The above argument, however, does not take into account the emission of eigenvalues. ${ }^{8}$ Even if the energy is not large enough to bind all $N$ eigenvalues, it may still be possible to

\footnotetext{
${ }^{7}$ Here we implicitly took a gauge in which the emitted D-brane is described by the $(N, N)$-components. Technical details about this gauge choice will be explained in section 3.2.1.

${ }^{8}$ The speculation that a subset of D-branes describes the small black hole existed for quite some time; see e.g. [21].
} 


\section{Black 3-brane}

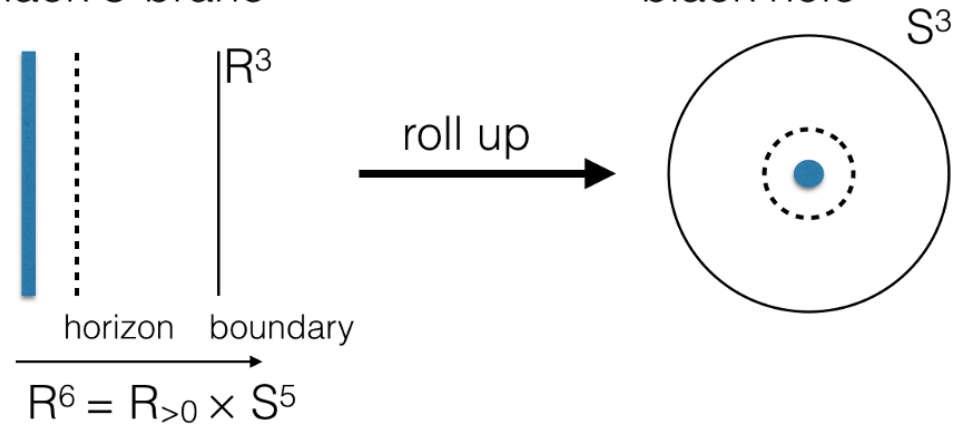

Figure 5. AdS black hole is obtained by rolling up black 3-brane.

bind $N_{\mathrm{BH}}$ eigenvalues, with $N_{\mathrm{BH}}<N$. Note that, because the space is compactified, there is no superselection of vacua; the value of the scalar field $X^{M}(M=1,2, \cdots, 6)$ should be determined dynamically, like in the Matrix Model of M-theory [12]. Also note that, unlike the Matrix Model of M-theory, this theory does not possess flat directions, because the scalars have a mass proportional to the inverse of the $S^{3}$ radius. Hence the emitted particles do not roll to infinity; they form a finite density gas and that can be absorbed again by the black hole. At some point, the emission and the absorption rates can balance.

We consider a state consisting of $\mathrm{BH}$ and gas in $4 \mathrm{~d} \mathcal{N}=4$ on $\mathbb{R}^{1} \times \mathrm{S}^{3}$. Our proposal is that a black hole consisting of $N_{\mathrm{BH}}$ eigenvalues and a gas consisting of $N_{\text {gas }}$ particles, where $N=N_{\mathrm{BH}}+N_{\text {gas }}$, can be described by matrices of the form ${ }^{9}$

$$
\left(\begin{array}{c|cccc}
X_{\mathrm{BH}}^{M} & & & & \\
\hline & x_{1}^{M} & & & \\
& & x_{2}^{M} & & \\
& & & \ddots & \\
& & & & x_{N_{\text {gas }}}^{M}
\end{array}\right),
$$

where $X_{\mathrm{BH}}$ is an $N_{\mathrm{BH}} \times N_{\mathrm{BH}}$ matrix.

Intuitively,

- In order for a bound state of eigenvalues (non-commutative block) to be formed, (almost) all off-diagonal elements must be excited, which costs a lot of energy.

- If the total energy of the system is big enough, say $E \sim N^{2}$, (almost) all off-diagonal elements can be excited. Hence $N=N_{\mathrm{BH}}, N_{\text {gas }}=0$ can be realized.

- If the energy is not that big, then $N_{\mathrm{BH}}$ becomes smaller. The number of degrees of freedom decreases dynamically, and the temperature of the system goes up. At some

\footnotetext{
${ }^{9}$ Here we implicitly took a gauge in which the 'black hole' comes to the upper-left corner. In section 3.2.1 we show how this gauge choice can be achieved, and that the gauge fixing and Faddeev-Popov terms are negligible. Strictly speaking, in addition to the elements shown in (3.1), there is some 'fuzziness' which describes short open strings stretched between nearby D-branes; see figure 7. Such a correction is negligible in the situations we consider below, where both $N_{\mathrm{BH}} / N$ is small but of order $N^{0}$.
} 


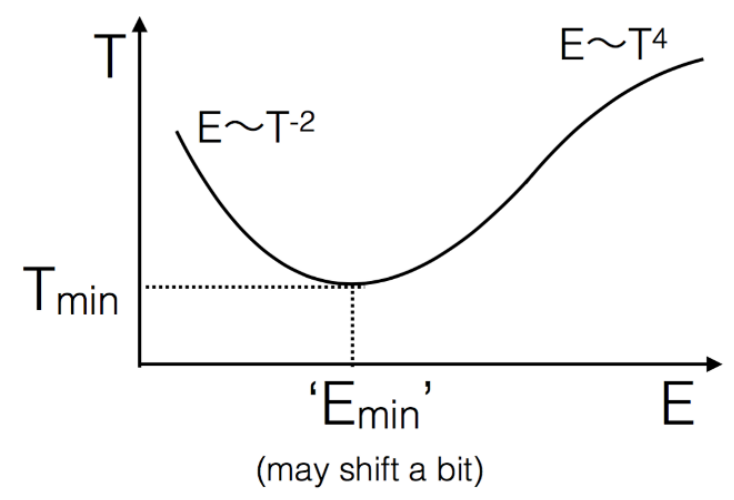

Figure 6. The microcanonical $E$-vs- $T$ phase diagram of $\mathrm{AdS}_{5}-\mathrm{BH}$ which is fills the $\mathrm{S}^{5}$ completely. $E<E_{\text {min }}$ does not have a counterpart in $4 \mathrm{~d} \mathrm{SYM}$; it is unstable with respect to the GregoryLaflamme instability along the $\mathrm{S}^{5}$.

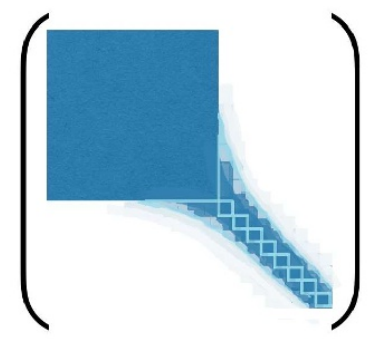

Figure 7. A more precise representation of the shape of the matrices describing a small black hole. In addition to the elements shown in (3.1), there is some 'fuzziness' which describes short open strings stretched between nearby D-branes. The light blue lines indicate where the blocks would be if the matrix were block diagonal. The color refers to the magnitude of the matrix elements, darker blue implying a larger magnitude.

point, (if the total energy is not too small) $N_{\mathrm{BH}}$ can become small enough so that off-diagonal elements in $X_{\mathrm{BH}}$ (number of degrees of freedom $\sim N_{\mathrm{BH}}^{2}$ ) can be excited.

- In the closed string picture: the string wants to become as long and winding as possible with the available amount of energy. It does this in order to gain more entropy. When the energy is not big enough, i.e. $N_{\mathrm{BH}}$ becomes too big, it ends up with a long but not very wound string. Then $N_{\mathrm{BH}}$ becomes smaller so that the string can take more complicated shapes.

Strictly speaking, there are strings connecting different gas-branes, and also the ones connecting the $\mathrm{BH}$ and gas-branes. See figure 7. Here we are assuming that most of them are long, heavy and do not play an important role.

In our calculation, we neglect the interaction between the emitted eigenvalues and the small BH, and treat it similarly as the 'large $\mathrm{BH}$ ' in the truncated $\mathrm{U}\left(N_{\mathrm{BH}}\right)$ gauge theory.

\subsubsection{Gauge fixing}

Here we explain how the (almost) block-diagonal form (3.1) and figure 7 can be obtained. Let us first introduce the maximally-diagonal gauge [27]. This gauge condition is based on 
the implicit assumption of the 'D-brane+open string' picture - diagonal elements are large, off-diagonal elements are small. However this is actually a gauge-dependent statement. Hence, we impose the condition that 'the matrices are as close to simultaneously diagonal as possible'. For that purpose we introduce $R_{i j}$ defined by

$$
R_{i j} \equiv \int d^{3} x \sum_{M=1}^{6}\left|X_{M, i j}\right|^{2} .
$$

Under the gauge transformation $X_{M} \rightarrow \Omega X_{M} \Omega^{-1}, R_{i j}$ transforms as

$$
R_{i j} \rightarrow R_{i j}(\Omega) \equiv \int d^{3} x \sum_{M=1}^{6}\left|\left(\Omega X_{M} \Omega^{-1}\right)^{i j}\right|^{2} .
$$

We choose $\Omega=\Omega_{\max }$ which maximizes $\operatorname{Tr} R=\sum_{i=1}^{N} R_{i i}(\Omega)$. Unless there are accidental degeneracies, such a $\Omega_{\max }$ is unique up to $\mathrm{U}(1)^{N-1}$ and simultaneous permutations of rows and columns. The $\tilde{X}_{M} \equiv \Omega_{\max } X_{M} \Omega_{\max }^{-1}$ are 'as close to simultaneously diagonal as possible'.

Now let us apply this gauge choice to the situation under consideration. Among the $N$ D-branes, $N_{\mathrm{BH}}$ form the small black hole. We assume both $N$ and $N_{\mathrm{BH}}$ are parametrically large, and $N_{\mathrm{BH}} / N$ is small but of order $N^{0}$. Then, the small black hole is stable, namely the value $N_{\mathrm{BH}}$ does not fluctuate much. The $N_{\mathrm{BH}}$ diagonal elements in the maximally diagonal gauge should then form a bunch (implying that the off-diagonal elements connecting them are not small), while other $N_{\text {gas }}=N-N_{\mathrm{BH}}$ diagonal elements are spread out. We can (partly) fix the ambiguity of the permutation of rows and columns by putting the $N_{\mathrm{BH}}$ size bunch in the upper-left corner of the matrices. Then the matrices takes the form (3.1).

In our arguments throughout the paper, intuitively, we are 'truncating' (or freezing out the gas degrees of freedom to reduce) $X$ to $\tilde{X}_{\mathrm{BH}}$. Note that this 'truncation' makes sense only in the microcanonical ensemble, and for a fixed value of the energy; when the extra energy is added, the bunch size increases in the original theory, while the bunch size is fixed and temperature increases in the truncated theory. The gauge fixing and the Faddeev-Popov terms associated with the maximally-diagonal gauge should be taken into account. But the maximally-diagonal gauge condition is rather nontrivial (note that $\Omega_{\max }$ is time-dependent!) and it is difficult to write down the gauge fixing and the FaddeevPopov terms. In the situation we have in mind, however, these terms are negligible up to the interaction with emitted particles. This is because, at each time $t$ the truncated action written by $\tilde{X}_{\mathrm{BH}}(t)$ is the same as the original action written by $X(t)$ to the leading order (i.e. $O\left(N^{2}\right)$ parts of the actions agree), as long as $N_{\mathrm{BH}} / N$ is of order $N^{0}$. As long as we consider physics of the small black hole (the bunch of $N_{\mathrm{BH}}$ D-branes), any gauge-equivalent profiles give simply the same path-integral weight as the truncated theory, to the leading order in $1 / N$.

Another way to fix the gauge is to introduce an 'external field'. Let $P_{n}$ be a projector to the $n \times n$ block, $P_{n}=\operatorname{diag}(1,1, \cdots, 1,0,0, \cdots, 0)$ with $n 1$ 's and $N-n 0$ 's. We can introduce an 'external field' which pushes BH to the upper-left corner, for example as

$$
S_{\text {ext }}=c \sum_{M} \operatorname{Tr}\left(X_{M}-P_{N_{\mathrm{BH}}} X_{M} P_{N_{\mathrm{BH}}}\right)^{2} .
$$


Note that this term manifestly breaks the full $\mathrm{SU}(N)$ symmetry. Then the $\mathrm{BH}$ is pushed to the upper-left corner by minimizing $S_{\text {ext }}$ which occurs at positive value $c$ which is small but $O\left(N^{0}\right)$. After taking $N \rightarrow \infty$, we turn off $c$. This is nothing but the usual prescription used for detecting spontaneous symmetry breaking.

\section{$3.310 d$ Schwarzschild at $1 / \lambda \ll N_{\mathrm{BH}} / N<1(\lambda \gg 1)$}

We will now make this scenario more precise. The AdS-Schwarzschild BH sitting at the origin of AdS corresponds to a bound state of eigenvalues around $X^{M}=0$.

The energy is

$$
E_{\text {total }}=E_{\mathrm{BH}}+E_{\text {gas }},
$$

and roughly speaking,

$$
E_{\mathrm{BH}} \sim N_{\mathrm{BH}}^{2}=O\left(N^{2}\right), \quad E_{\mathrm{gas}} \sim\left(N-N_{\mathrm{BH}}\right)=O(N) .
$$

Hence we ignore $E_{\text {gas }}$.

We have to determine $N_{\mathrm{BH}}$ for given $E \simeq E_{\mathrm{BH}}<E_{\min }\left(N, g_{\mathrm{YM}}^{2}\right)$. Let us firstly give a heuristic gauge theory argument which does not rely on the gravity dual. Suppose the action is dominated by the $\frac{N}{\lambda} \operatorname{Tr}\left[X_{M}, X_{M^{\prime}}\right]^{2}$-term, which should be true at strong coupling. Note that the coupling $\lambda$ disappears in terms of $X^{\prime} \equiv \lambda^{-1 / 4} X$; hence the eigenvalues of $X^{\prime}$ are of order 1 , and the eigenvalues of $X$ scale as $\lambda^{1 / 4}$ when $g_{\mathrm{YM}}^{2}$ is varied. When the bunch size decreases to $N_{\mathrm{BH}}$, the radius of the bunch scales as $\lambda_{\mathrm{BH}}^{1 / 4}$, assuming the interactions with the emitted branes do not affect the size of the bunch significantly. Here $\lambda_{\mathrm{BH}}=g_{\mathrm{YM}}^{2} N_{\mathrm{BH}}=\frac{N_{B H}}{N} \lambda$, and we assumed $\lambda_{\mathrm{BH}} \gg 1$. It would be natural ${ }^{10}$ to expect that the typical energy scale is set by the inverse of the eigenvalue distribution, $\lambda_{\mathrm{BH}}^{-1 / 4}$. Then, combined with the fact that the large $\mathrm{BH}$ should have $T_{\min }\left(N, g_{\mathrm{YM}}^{2}\right) \sim 1$, we obtain ${ }^{11}$

$$
T_{\min }\left(N_{\mathrm{BH}}, g_{\mathrm{YM}}^{2}\right) \sim\left(\lambda_{\mathrm{BH}} / \lambda\right)^{-1 / 4} \sim \alpha^{-1 / 4}, \quad \alpha \equiv \frac{N_{\mathrm{BH}}}{N},
$$

and combined with the 't Hooft counting and $E_{\min }\left(N, g_{\mathrm{YM}}^{2}\right) \sim N^{2}$,

$$
E_{\min }\left(N_{\mathrm{BH}}, g_{\mathrm{YM}}^{2}\right) \sim N_{\mathrm{BH}}^{2}\left(\lambda_{\mathrm{BH}} / \lambda\right)^{-1 / 4}=N^{2} \alpha^{7 / 4}, \quad S_{\min }\left(N_{\mathrm{BH}}, g_{\mathrm{YM}}^{2}\right) \sim N_{\mathrm{BH}}^{2}=N^{2} \alpha^{2} .
$$

A better argument inspired by string theory goes as follows. In the dual gravity picture, the 'smallest large black hole' is a bunch of eigenvalues filling $\mathrm{AdS}_{5}$ almost completely. In the D-brane picture, $\mathrm{AdS}_{5}$ is made of $\mathbb{R}^{1} \times \mathrm{S}^{3}$ and $\mathbb{R}_{>0}$, where $\mathbb{R}_{>0}$ is the radial coordinate of the transverse $\mathbb{R}^{6}$. Intuitively, the boundary $\mathrm{S}^{3}$ is almost touching the D-branes. When the bunch shrinks, the radius becomes smaller by a factor of $\left(\lambda_{\mathrm{BH}} / \lambda\right)^{1 / 4}$. In order to measure the energy of this bunch, we imagine a sphere right outside of the bunch, and consider

\footnotetext{
${ }^{10}$ The existence of another scale $R_{\mathrm{S}^{3}}=1$ makes the situation subtle. When $\left(\lambda_{\mathrm{BH}} / \lambda\right)^{-1 / 4} \gg 1$, the two energy scales $\left(\lambda_{\mathrm{BH}} / \lambda\right)^{-1 / 4}$ and $R_{\mathrm{S}^{3}}=1$ are clearly separated. Then energy scale should be dominated by $\left(\lambda_{\mathrm{BH}} / \lambda\right)^{-1 / 4}$ which is the size of the eigenvalue distribution.

${ }^{11}$ We would like to thank Juan Maldacena and Kostas Skenderis for pointing out several miscalculations in the first version of this paper, including this part. It helped us debug a few wrong points in the argument.
} 
only the interactions between D-branes and open strings inside this sphere. If we further decrease the energy inside this restricted region, the bunch becomes smaller. If we pump up the energy, the bunch becomes hotter. (Without this restriction, the temperature does not have to go up because the bunch can become larger.) Hence, with this restriction, the bunch is 'the smallest possible large black hole'. Note that this restriction is natural from the gauge theory, or 'open string', point of view, in which the gravitational back-reaction is not included. From the dual gravity theory point of view ('closed string picture' in the sense of usual open string/closed string duality), a naive truncation near the horizon is very problematic. As we will see shortly, the counterpart of this restriction on the gravity side is something different; the curvature radius changes as well on the gravity side. Mapping back to the gauge theory, the energy of this bunch should be described by the fully noncommutative phase of $\mathrm{U}\left(N_{\mathrm{BH}}\right)$ gauge theory with $R_{\mathrm{S}^{3}}=\left(\lambda_{\mathrm{BH}} / \lambda\right)^{1 / 4}$; here we identified the $\mathrm{S}^{3}$ with the surface right outside the bunch. ${ }^{12}$ This scaling of $R_{\mathrm{S}^{3}}$ naturally suggests the scalings in (3.8). Note that the 't Hooft coupling changes from $\lambda=g_{\mathrm{YM}}^{2} N$ to $\lambda_{\mathrm{BH}}=g_{\mathrm{YM}}^{2} N_{\mathrm{BH}}$; this does not affect the result because the quantities of interest do not explicitly depend on the 't Hooft coupling.

In order to calculate the energy, entropy, and justify (3.8) more quantitatively, let us appeal to the AdS/CFT duality for large BH from here on, and go to the gravity picture. If we assume the dual gravity calculation of the large BH to be correct, ${ }^{13}$ when $\lambda=g_{\mathrm{YM}}^{2} N \gg 1$, the temperature of $\mathrm{AdS}_{5}$ black hole is given by

$$
T_{\mathrm{AdS}-\mathrm{BH}}=\frac{2 r_{+}^{2}+R_{\mathrm{AdS}}^{2}}{2 \pi R_{\mathrm{AdS}}^{2} r_{+}},
$$

which is minimized at $2 r_{+}^{2}=R_{\mathrm{AdS}}^{2}=1$,

$$
T_{\min }\left(N, g_{\mathrm{YM}}^{2}\right)=\frac{\sqrt{2}}{\pi R_{\mathrm{AdS}}} .
$$

The area of the horizon is $2 \pi^{2} r_{+}^{3}$, and hence the entropy is $S_{\text {AdS-BH }}=\pi^{2} r_{+}^{3} /\left(2 G_{5, \mathrm{~N}}\right)$. Here $G_{5, \mathrm{~N}}$ is the $5 \mathrm{~d}$ Newton constant, which is related to the $10 \mathrm{~d}$ Newton constant ${ }^{14}$ $G_{10, \mathrm{~N}} \sim 1 / N^{2}$, by $G_{10, \mathrm{~N}}=G_{5, \mathrm{~N}} \cdot\left(\pi^{3} R_{\mathrm{AdS}}^{5}\right)$, where the denominator $\pi^{3} R_{\mathrm{AdS}}^{5}$ is the area of the $\mathrm{S}^{5}$. Hence

$$
S=\frac{r_{+}^{3} \pi^{5} R_{\mathrm{AdS}}^{5}}{2 G_{10, \mathrm{~N}}}
$$

By using $d E=T d S$, we obtain

$$
E=\frac{3 \pi^{4} R_{\mathrm{AdS}}^{3}}{8 G_{10, \mathrm{~N}}}\left(r_{+}^{4}+R_{\mathrm{AdS}}^{2} r_{+}^{2}\right) .
$$

\footnotetext{
${ }^{12}$ As mentioned above, if this rescaling is not performed, then the number of D-branes in the bunch (i.e. $N_{\mathrm{BH}}$ ) can change as the energy grows, and hence the identification with the 'smallest large black hole' fails.

${ }^{13}$ This is the only assumption which relies on the dual gravity description. Note that we assumed here the validity of the dual gravity description for the large black hole in order to derive (3.9)-(3.13) and will derive the energy of the small black hole in the following.

${ }^{14}$ This is different from usual value in the Einstein frame, $G_{10, \mathrm{~N}} \sim \lambda^{2} / N^{2}$, because we are using the dual frame.
} 
$E_{\min }\left(N, g_{\mathrm{YM}}^{2}\right)$ and $S_{\min }\left(N, g_{\mathrm{YM}}^{2}\right)$ are given by using $r_{+}^{2} \sim R_{\mathrm{AdS}}^{2}=1$ and we obtain

$$
E_{\min }\left(N, g_{\mathrm{YM}}^{2}\right) \sim \frac{R_{\mathrm{AdS}}^{7}}{G_{10, \mathrm{~N}}}, \quad S_{\min }\left(N, g_{\mathrm{YM}}^{2}\right) \sim \frac{R_{\mathrm{AdS}}^{8}}{G_{10, \mathrm{~N}}} .
$$

When the bunch shrinks, $R_{\text {AdS }}=1$ should be replaced by ${ }^{15} R_{\text {AdS }}^{\prime}=\left(N_{\mathrm{BH}} / N\right)^{1 / 4}$ associated with the rescaling of $R_{\mathrm{S}^{3}}$. The Newton constant remains unchanged, because the rescaling factors associated with $R_{\mathrm{AdS}} \rightarrow R_{\mathrm{AdS}}^{\prime}$ and $N \rightarrow N_{\mathrm{BH}}$ cancel with each other. Hence we should have (3.8) again. ${ }^{16}$

We identify the energy and entropy of the small black hole with these values:

$$
E_{\mathrm{BH}}=E_{\min }\left(N_{\mathrm{BH}}, g_{\mathrm{YM}}^{2}\right), \quad S_{\mathrm{BH}}=S_{\min }\left(N_{\mathrm{BH}}, g_{\mathrm{YM}}^{2}\right) .
$$

Then,

$$
T_{\mathrm{BH}}=\frac{d E_{\mathrm{BH}}}{d S_{\mathrm{BH}}} \sim \alpha^{-1 / 4} .
$$

Note that this might be different from $T_{\min }\left(N_{\mathrm{BH}}, g_{\mathrm{YM}}^{2}\right)$. By substituting (3.15) into (3.8), we obtain

$$
E_{\mathrm{BH}} \sim \frac{N^{2}}{T_{\mathrm{BH}}^{7}} \sim \frac{1}{G_{10, \mathrm{~N}} T_{\mathrm{BH}}^{7}} .
$$

Before closing this section, let us give a comment on a confusing point associated with the evaluation of (3.8) via the $\mathrm{U}\left(N_{\mathrm{BH}}\right)$ theory. When $N_{\mathrm{BH}}$ decreases, if one naively 'truncated' the theory to the $\mathrm{U}\left(N_{\mathrm{BH}}\right)$ theory without rescaling $R_{\mathrm{S}^{3}}$, one would not have an $N_{\mathrm{BH}}$ dependence. This treatment is wrong because the truncation and the variation of the energy do not commute. When energy is added, in the original theory $N_{\mathrm{BH}}$ increases and as a result the temperature can go down, while in the truncated theory $N_{\mathrm{BH}}$ cannot change and the temperature has to go up. The argument on the scaling of eigenvalues, which is provided at the beginning of this section, may seem to suffer from the same subtlety. However, we do not find a problem there, because we did not change the energy, rather we varied $g_{\mathrm{YM}}^{2}$. The result (3.8) does not explicitly depend on $g_{\mathrm{YM}}^{2}$; namely the bunch size is determined solely by the energy.

\subsubsection{The phase transition from the big black hole to the small black hole}

According to the calculation in the gravity side [4], the transition from the large black hole to the small black hole is of first order, and the small black hole is hotter than the large black hole at the same energy. (More precisely speaking, the large BH becomes a lumpy black hole [28], and then becomes unstable.)

In our gauge theory argument, the order of the transition is not clear. However, if we assume the transition is of first order, then the small black hole must be hotter, due to the Higgsing.

\footnotetext{
${ }^{15}$ Because the curvature radius changes, this is different from the 'truncation' of the geometry.

${ }^{16}$ This kind of matching has a flavor similar to the correspondence principle [18, 22].
} 


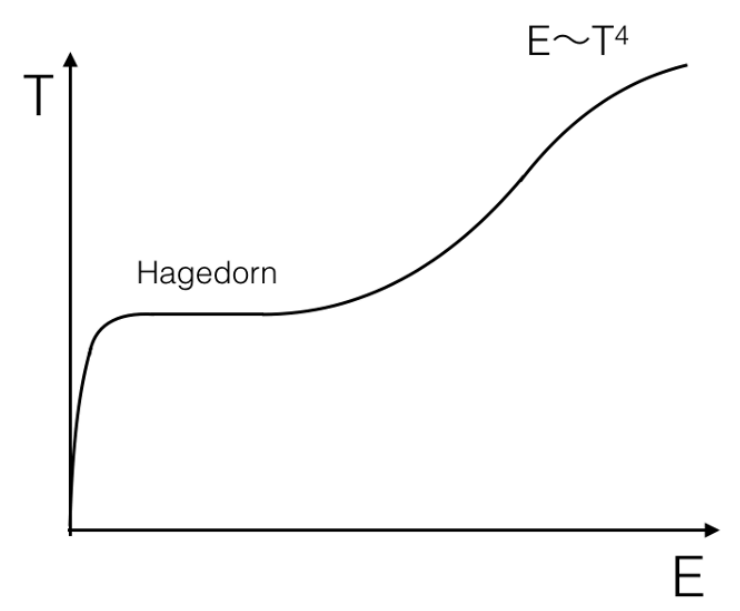

Figure 8. Phase diagram of the weakly coupled 4d SYM, $\lambda \ll 1$. The difference from the one at $\lambda \gg 1$ (figure 1) is not just an overall factor.

\subsection{The Hagedorn growth at $N_{\mathrm{BH}} / N \lesssim 1 / \lambda(\lambda \gg 1)$}

In the previous section, we have assumed $\lambda_{\mathrm{BH}} \equiv g_{\mathrm{YM}}^{2} N_{\mathrm{BH}} \gg 1$. When $\lambda_{\mathrm{BH}} \lesssim 1$, we can use perturbation theory. There, $T_{\min }$, which is of same order as the deconfining and hagedorn temperatures, is of $\mathrm{O}\left(N^{0}\right)$ and goes to a $\lambda_{\mathrm{BH}}$-independent constant. The growth of the temperature stops when $N_{\mathrm{BH}} / N \sim 1 / \lambda$, at $T_{\mathrm{BH}} \sim 1, E / N^{2} \sim 1 / \lambda^{2}$. The constant$T$ behavior below this point looks like the Hagedorn behavior; actually, in the closed string picture, the length of the long string $N_{\mathrm{BH}}^{2}$ increases with $E$. This is exactly the Hagedorn behavior! The energy $E$ and the entropy $S(E)$ are proportional to each other, $E_{\mathrm{BH}} \sim S_{\mathrm{BH}} \sim N_{\mathrm{BH}}^{2}$. In other words, when energy is added, it is used for exciting more matrix degrees of freedom, rather than increasing the energy per degree of freedom.

Note also that, at $N_{\mathrm{BH}} / N \sim 1 / \lambda$, the energy becomes $E \sim 1 /\left(l_{\mathrm{S}}^{7} G_{10, \mathrm{~N}}\right)$, which is the endpoint of the Hagedorn growth expected on the gravity side [3].

\subsection{The case of weak coupling $\lambda \ll 1$}

The same idea of emission of eigenvalues can be applied to the weakly coupled region of $4 \mathrm{~d}$ SYM. In this region the Hagedorn growth continues until $N_{\mathrm{BH}}$ reaches $N$, and hence a negative specific heat is not expected; see figure 8 . This region is rather different from the strong coupling region (figure 1). The difference is not just a factor $3 / 4$ rather the shape is different.

This phase diagram figure 8 has been known for quite some time, see e.g. [29]. We have just rephrased the known result, in order to show the consistency of our proposal.

\section{6 $\quad \mathrm{SO}(6)$ breaking}

The $10 \mathrm{~d} \mathrm{BH}$ is localized on the $\mathrm{S}^{5}$. Hence the $\mathrm{SO}(6)$ rotational symmetry should be broken. A natural possibility would be that the 'smallest possible large $\mathrm{BH}$ ' is something like a lumpy $\mathrm{BH}[28]$ which breaks $\mathrm{SO}(6)$ and the eigenvalue distribution in gauge theory side also breaks $\mathrm{SO}(6)$. More analysis is desirable concerning this point. 


\subsection{Other topologies}

Let us consider other topologies such as $\mathrm{S}^{2} \times \mathrm{S}^{1}$ and $\mathrm{T}^{3}=\mathrm{S}^{1} \times \mathrm{S}^{1} \times \mathrm{S}^{1} \cdot{ }^{17,18}$ The important difference here is that $\mathrm{S}^{1}$ is not intrinsically curved. For this reason, the circumference of the $\mathrm{S}^{1}$ does not necessarily introduce a 'scale' to the setup under consideration; indeed, as long as the center symmetry along the $\mathrm{S}^{1}$ is unbroken, translational invariant quantities such as the energy density is independent of the circumference of the $\mathrm{S}^{1}$, due to the Eguchi-Kawai equivalence [30].

Let us consider $4 \mathrm{~d}$ SYM on $\mathrm{T}^{3}$ first. The crucial difference from $\mathrm{S}^{3}$ and $\mathrm{S}^{2} \times \mathrm{S}^{1}$ compactifications is that D3-branes wrapping on three-torus have Ramond-Ramond charge. Therefore, this theory does not describe a Schwarzschild black hole. ${ }^{19}$ There is a $\mathbb{Z}_{N}^{3}$ center symmetry, which can be broken at small volume. When $\mathbb{Z}_{N}^{p} \subset \mathbb{Z}_{N}^{3}$ is unbroken, the theory is approximated by $(p+1)$-d SYM on $\mathbb{R}^{1, p}$, due to the dimensional reduction along the directions where $\mathbb{Z}_{N}$ is broken and the Eguchi-Kawai equivalence along the directions where $\mathbb{Z}_{N}$ is unbroken. The gravity side is described by the black $p$-brane in the Tdual picture. In the gravity side, the center-breaking phase transitions are interpreted as the black hole/black string phase transition [25] and its higher-dimensional analogues; for details, see [31-34].

Next let us consider $4 \mathrm{~d} \mathrm{SYM}$ on $\mathrm{S}^{2} \times \mathrm{S}^{1}$. We take the radius of the two-sphere to be 1 . Let us fix the circumference of $\mathrm{S}^{1}$, which we denote by $L$, and increase the energy $E$ from zero. When $E$ is sufficiently large, the center symmetry is unbroken. When the energy density per length $\mathcal{E}_{\mathrm{BS}}$ is fixed and $L$ is changed, the Eguchi-Kawai equivalence guarantees the $L$-independence of the system. In gravity side, the topology of the small black hole becomes $S^{7} \times \mathbb{R}$ as the circumference of the $S^{1}$ goes to infinity. Hence they behave as a black string (BS), rather than a black hole. ${ }^{20}$ At larger $L, \mathcal{E}_{\mathrm{BS}}$ can be made smaller without breaking the center symmetry. There $\mathcal{E}_{\mathrm{BS}}$ should behave in the same way as a $9 \mathrm{~d}$ black hole,

$$
\mathcal{E}_{\mathrm{BS}} \sim \frac{1}{G_{10, \mathrm{~N}} T_{\mathrm{BS}}^{6}}
$$

This can be derived from gauge theory as follows. Firstly, the scaling of the eigenvalues do not change, and hence $\alpha=N_{\mathrm{BS}} / N$ should be related to the temperature as

$$
T_{\min }\left(N_{\mathrm{BS}}, g_{\mathrm{YM}}^{2}\right) \sim\left(\lambda_{\mathrm{BS}} / \lambda\right)^{-1 / 4} \sim \alpha^{-1 / 4}, \quad \alpha \equiv \frac{N_{\mathrm{BS}}}{N} .
$$

Here, $N_{\mathrm{BS}}$ is the same as $N_{\mathrm{BH}}$ we used before; we just emphasized that this is related to the black string. Combined with the 't Hooft counting, the dimensional analysis and

\footnotetext{
${ }^{17}$ We thank our anonymous referee for suggesting to study these examples.

${ }^{18}$ We impose a periodic boundary condition along $\mathrm{S}^{1}$.

${ }^{19}$ Outside of the 't Hooft limit, it is possible to take the limit describing the M-theory regime of the Matrix Model of M-theory, where the 11d Schwarzschild black hole may emerge. We do not consider this limit in this paper.

${ }^{20}$ In the T-dual picture, we find D2-branes smeared along the T-dual circle, which also can be interpreted as a black string.
} 
$\mathcal{E}_{\text {min }}\left(N, g_{\mathrm{YM}}^{2}\right) \sim N^{2}, \mathcal{S}_{\min }\left(N, g_{\mathrm{YM}}^{2}\right) \sim N^{2}$, it leads to

$$
\begin{aligned}
& \mathcal{E}_{\min }\left(N_{\mathrm{BS}}, g_{\mathrm{YM}}^{2}\right) \sim N_{\mathrm{BS}}^{2}\left(\lambda_{\mathrm{BS}} / \lambda\right)^{-2 / 4}=N^{2} \alpha^{6 / 4}, \\
& \mathcal{S}_{\min }\left(N_{\mathrm{BS}}, g_{\mathrm{YM}}^{2}\right) \sim N_{\mathrm{BS}}^{2}\left(\lambda_{\mathrm{BS}} / \lambda\right)^{-1 / 4}=N^{2} \alpha^{7 / 4} .
\end{aligned}
$$

Hence (3.17) naturally follows.

When the energy is small, then the center symmetry breaks. On the gravity side, in the T-dual picture, the center breaking can be interpreted as a topology change from the smeared D2-branes (BS) to the localized D2-branes (BH). Note that this transition is rather different from the localization along the $\mathrm{S}^{5}$ which we have discussed in the previous sections; the topology change takes place only in the T-dual picture. When the energy is sufficiently small, 4d SYM reduces to $3 \mathrm{~d}$ SYM on $\mathrm{S}^{2}$. Then the argument of section 4.2 can be applied. Near the transition point $E=E_{c}$, the analytic treatment is difficult; however, previous studies in analogous situations (e.g. [35]) suggests that the expansion with respect to $E / E_{c}$ works well.

\section{Other cases}

\subsection{ABJM theory and 11d black hole}

The argument shown above can be applied to other quantum field theories as well. As an example, let us consider the M-theory region of the ABJM theory [36] on $\mathbb{R}^{1} \times \mathrm{S}^{2}$. Namely we consider the Chern-Simons level $k=1$, 't Hooft coupling $\lambda=N / k=N$. The gravity dual is M-theory on $\mathrm{AdS}_{4} \times \mathrm{S}^{7}$. In this case, the microscopic picture on the gravity side is not clear, other than that $N$ corresponds to the number of M2-branes. However it would be natural to assume that the small $\mathrm{BH}$ is described in the same way as in $4 \mathrm{~d} \mathcal{N}=4 \mathrm{SYM}$, by a bunch of $N_{\mathrm{BH}} \mathrm{M} 2$-branes, and the calculation goes through in the same manner.

With this assumption, by using $R_{\mathrm{AdS}} \sim(k N)^{1 / 6} l_{\mathrm{P}}=N^{1 / 6} l_{\mathrm{P}}$ and $G_{11, \mathrm{~N}} \sim l_{\mathrm{P}}^{9}$ where $G_{11, \mathrm{~N}}$ and $l_{\mathrm{P}}^{9}$ are the eleven-dimensional Newton constant and Planck scale, respectively, the energy and entropy are estimated $a^{21}$

$$
E_{\mathrm{min}} \sim \frac{R_{\mathrm{AdS}}}{G_{4, \mathrm{~N}}} \sim \frac{R_{\mathrm{AdS}}^{8}}{G_{11, \mathrm{~N}}} \sim \frac{N^{4 / 3}}{l_{\mathrm{P}}}, \quad E_{\mathrm{BH}} \sim \frac{N_{\mathrm{BH}}^{4 / 3}}{l_{\mathrm{P}}} .
$$

and

$$
S_{\min } \sim \frac{R_{\mathrm{AdS}}^{2}}{G_{4, \mathrm{~N}}} \sim \frac{R_{\mathrm{AdS}}^{9}}{G_{11, \mathrm{~N}}} \sim N^{3 / 2}, \quad S_{\mathrm{BH}} \sim N_{\mathrm{BH}}^{3 / 2}
$$

Therefore,

$$
T_{\mathrm{BH}}=\frac{d E_{\mathrm{BH}}}{d S_{\mathrm{BH}}} \sim N_{\mathrm{BH}}^{-1 / 6} / l_{\mathrm{P}},
$$

\footnotetext{
${ }^{21}$ For $4 \mathrm{~d} \mathcal{N}=4 \mathrm{SYM}$, we used the fact that the eigenvalues scales as $\lambda^{1 / 4}$. The fact that this scales in the same manner $R_{\text {AdS }}$ is important. Here, we need to use a similar relation: the bifundamental scalars $\phi$, which describes the moduli space of M2-branes, should scale as $N^{1 / 6}$. In the 't Hooft limit $\left(N / k=O\left(N^{0}\right)\right)$, because $\phi$ has a potential of the form $N \phi^{6} / \lambda$, the scaling should be $\lambda^{1 / 6}=(N / k)^{1 / 6}$. The same behavior is expected in the M-theory region, up to $1 / N$-suppressed corrections [37].
} 
yielding finally

$$
E_{\mathrm{BH}} \sim \frac{1}{G_{11, \mathrm{~N}} T_{\mathrm{BH}}^{8}}
$$

This correctly reproduces the property of the eleven-dimensional Schwarzschild black hole. In this case (ABJM, $k=1$ ) the system is strongly coupled even for small $N_{\mathrm{BH}}$, and hence the Hagedorn behavior discussed in section 3.4 does not set in.

\subsection{More generic theories}

The same power counting will hold for other theories as well, including non-conformal theories, if they possess a similar matrix description. We assume that the geometry consists of $d$ non-compact and $D-d$ compact dimensions (where $D=10$ and $D=11$ for theories with respectively string and M-theory duals). The space-time does not necessarily have to be a product like $\mathrm{AdS} \times \mathrm{X}$ (where $\mathrm{X}$ is a compact manifold), as long as the notion of large and small black holes still makes sense. Furthermore, suppose there is only one typical length scale $R$ in the dual geometry, like $R_{\text {AdS }}$. Let us also assume that the eigenvalues and $R$ scale in the same manner as a function of the coupling constant. Then, it is natural to expect that the minimum energy and temperature are obtained by the dimensional analysis as

$$
E_{\min } \sim \frac{R^{D-3}}{G_{D, \mathrm{~N}}}, \quad S_{\min } \sim \frac{R^{D-2}}{G_{D, \mathrm{~N}}}
$$

By going to the dual field theory description and applying the same argument for the small black hole, we obtain

$$
E_{\mathrm{BH}} \sim \frac{(R \alpha)^{D-3}}{G_{D, \mathrm{~N}}}, \quad S_{\mathrm{BH}} \sim \frac{(R \alpha)^{D-2}}{G_{D, \mathrm{~N}}}
$$

and

$$
T_{\mathrm{BH}} \sim 1 /(R \alpha)
$$

where $\alpha=\left(N_{\mathrm{BH}} / N\right)^{p}$. The power $p$ may depend on the theory. This gives the expected scaling,

$$
E_{\mathrm{BH}} \sim \frac{1}{G_{D, \mathrm{~N}} T_{\mathrm{BH}}^{D-3}}, \quad S_{\mathrm{BH}} \sim \frac{1}{G_{D, \mathrm{~N}} T_{\mathrm{BH}}^{D-2}} .
$$

\section{Discussions}

A rather striking consequence of our proposal is that the small $\mathrm{BH}$ is essentially like a large $\mathrm{BH}$ from the point of view of the gauge theory; it is the "smallest possible large $\mathrm{BH}$ ', which is continuously connected to the high- $T$ region. Seen from gauge theory, it is simply a thermal state, but with different 'matrix size'. Hence the study of the large BH provides us with important lessons on the small BH. Another lesson is the importance of 
eigenvalue dynamics in the gauge/gravity duality a la Maldacena. Although the importance of eigenvalue dynamics has been widely appreciated in the 20th century, for example in the Matrix Theory conjecture [12], it has somehow almost been forgotten after the Maldacena conjecture (AdS/CFT). It is, however, an important piece for understanding black hole evaporation, even in the context of the Maldacena conjecture, as emphasized in this paper and refs. [16, 17]. Due to this, a detailed study of eigenvalue dynamics should help lead us to an understanding of the bulk geometry, including the horizon of the black hole. Note that the large $\mathrm{BH}$ can be studied by using the canonical ensemble, which makes the numerical simulation rather straightforward with the Matsubara formalism. It would provide us with a first-principle study of the geometric structure of the Schwarzschild black hole based on gauge theory. Such a study should be important for various problems associated with the black hole information puzzle.

More tests would be desirable to establish the proposal more rigidly. One interesting and doable direction would be a consistency check based on dual gravity calculations. If our proposal is correct, the small black hole and the large black hole at ' $E_{\min }$ ' describe essentially the same dual gauge theory, up to the rescaling of the 't Hooft coupling associated with the emission of D-branes. Recently, dual gravity calculations for the small black hole have been performed in $[4,26]$. There is also an attempt for studying the small $\mathrm{AdS}_{5}$ black hole, which is not localized along $S^{5}$ which can be found in [38]. Since the agreement should become better when 10d black hole is smaller, such tests might be doable without relying on the very hard numerical calculations needed to include the finite-size effects [4, 26]. We have not yet understood how the breakdown of $\mathrm{SO}(6)$ symmetry can be seen in terms of eigenvalues. It would be nice if we could make progress in near future.

In this paper we truncated large matrices to small matrices. More refined treatments, for example something like the matrix renormalization group [39] which integrates out the emitted eigenvalues, would allow us to extract more information about black holes.

\section{Acknowledgments}

We would like to thank Leonard Susskind for the collaboration toward the end of the project. As explained in the introduction, he had essentially the same idea as ours. We would also like to thank Juan Maldacena and Kostas Skenderis for pointing out miscalculations in the first version of this paper, which helped us correcting a few wrong arguments. Furthermore we thank Ahmed Almheiri, Tom Banks, Evan Berkowitz, Oscar Dias, Ethan Dyer, Blaise Goutéraux, Guy Gur-Ari, Daniel Harlow, Joao Penedones, Enrico Rinaldi, Jorge Santos, Andreas Schäfer, Stephen Shenker, David Tong and Ying Zhao for discussions and comments. In particular, $O(30)$ email exchanges with Daniel Harlow within two days certainly helped us improving the presentation. The work of M.H. is supported in part by the Grant-in-Aid of the Japanese Ministry of Education, Sciences and Technology, Sports and Culture (MEXT) for Scientific Research (No. 25287046). The work of J.M. is supported by the California Alliance fellowship (NSF grant 32540). 
Open Access. This article is distributed under the terms of the Creative Commons Attribution License (CC-BY 4.0), which permits any use, distribution and reproduction in any medium, provided the original author(s) and source are credited.

\section{References}

[1] J.M. Maldacena, The Large-N limit of superconformal field theories and supergravity, Int. J. Theor. Phys. 38 (1999) 1113 [hep-th/9711200] [InSPIRE].

[2] E. Witten, Anti-de Sitter space and holography, Adv. Theor. Math. Phys. 2 (1998) 253 [hep-th/9802150] [INSPIRE].

[3] O. Aharony, S.S. Gubser, J.M. Maldacena, H. Ooguri and Y. Oz, Large-N field theories, string theory and gravity, Phys. Rept. 323 (2000) 183 [hep-th/9905111] [INSPIRE].

[4] Ó.J.C. Dias, J.E. Santos and B. Way, Localised $A d S_{5} \times S^{5}$ Black Holes, Phys. Rev. Lett. 117 (2016) 151101 [arXiv: 1605.04911] [INSPIRE].

[5] E. Berkowitz, E. Rinaldi, M. Hanada, G. Ishiki, S. Shimasaki and P. Vranas, Precision lattice test of the gauge/gravity duality at large- $N$, Phys. Rev. D 94 (2016) 094501 [arXiv: 1606.04951] [INSPIRE].

[6] A.V. Smilga, Comments on thermodynamics of supersymmetric matrix models, Nucl. Phys. B 818 (2009) 101 [arXiv:0812.4753] [INSPIRE].

[7] T. Wiseman, On black hole thermodynamics from super Yang-Mills, JHEP 07 (2013) 101 [arXiv: 1304.3938] [INSPIRE].

[8] T. Morita and S. Shiba, Thermodynamics of black M-branes from SCFTs, JHEP 07 (2013) 100 [arXiv: 1305. 0789] [INSPIRE].

[9] T. Morita, S. Shiba, T. Wiseman and B. Withers, Moduli dynamics as a predictive tool for thermal maximally supersymmetric Yang-Mills at large-N, JHEP 07 (2015) 047 [arXiv: 1412.3939] [INSPIRE].

[10] N. Itzhaki, J.M. Maldacena, J. Sonnenschein and S. Yankielowicz, Supergravity and the large-N limit of theories with sixteen supercharges, Phys. Rev. D 58 (1998) 046004 [hep-th/9802042] [INSPIRE].

[11] E. Witten, Bound states of strings and p-branes, Nucl. Phys. B 460 (1996) 335 [hep-th/9510135] [INSPIRE].

[12] T. Banks, W. Fischler, S.H. Shenker and L. Susskind, $M$ theory as a matrix model: A Conjecture, Phys. Rev. D 55 (1997) 5112 [hep-th/9610043] [InSPIRE].

[13] B. de Wit, J. Hoppe and H. Nicolai, On the quantum mechanics of supermembranes, Nucl. Phys. B 305 (1988) 545 [INSPIRE].

[14] P. Kraus, Lectures on black holes and the $A d S_{3} / C F T_{2}$ correspondence, Lect. Notes Phys. 755 (2008) 193 [hep-th/0609074] [INSPIRE].

[15] T. Banks, W. Fischler, I.R. Klebanov and L. Susskind, Schwarzschild black holes from matrix theory, Phys. Rev. Lett. 80 (1998) 226 [hep-th/9709091] [InSPIRE].

[16] E. Berkowitz, M. Hanada and J. Maltz, Chaos in Matrix Models and Black Hole Evaporation, Phys. Rev. D 94 (2016) 126009 [arXiv: 1602.01473] [INSPIRE]. 
[17] E. Berkowitz, M. Hanada and J. Maltz, A microscopic description of black hole evaporation via holography, Int. J. Mod. Phys. D 25 (2016) 1644002 [arXiv:1603.03055] [INSPIRE].

[18] L. Susskind, Some speculations about black hole entropy in string theory, hep-th/9309145 [INSPIRE].

[19] M. Hanada, J. Maltz and L. Susskind, Deconfinement transition as black hole formation by the condensation of QCD strings, Phys. Rev. D 90 (2014) 105019 [arXiv:1405.1732] [INSPIRE].

[20] J.B. Kogut and L. Susskind, Hamiltonian Formulation of Wilson's Lattice Gauge Theories, Phys. Rev. D 11 (1975) 395 [inSPIRE].

[21] C.T. Asplund and D. Berenstein, Small AdS black holes from SYM, Phys. Lett. B 673 (2009) 264 [arXiv:0809.0712] [INSPIRE].

[22] G.T. Horowitz and J. Polchinski, A Correspondence principle for black holes and strings, Phys. Rev. D 55 (1997) 6189 [hep-th/9612146] [INSPIRE].

[23] S. Catterall, P.H. Damgaard, T. Degrand, R. Galvez and D. Mehta, Phase Structure of Lattice $N=4$ Super Yang-Mills, JHEP 11 (2012) 072 [arXiv:1209.5285] [INSPIRE].

[24] D. Schaich and S. Catterall, Maximally supersymmetric Yang-Mills on the lattice, in proceedings of the Sakata Memorial KMI Workshop on Origin of Mass and Strong Coupling Gauge Theories (SCGT15), Nagoya, Japan, 3-6 March 2015 [arXiv:1508.00884] [INSPIRE].

[25] R. Gregory and R. Laflamme, Black strings and p-branes are unstable, Phys. Rev. Lett. 70 (1993) 2837 [hep-th/9301052] [INSPIRE].

[26] V.E. Hubeny and M. Rangamani, Unstable horizons, JHEP 05 (2002) 027 [hep-th/0202189] [INSPIRE].

[27] T. Azeyanagi, M. Hanada, T. Hirata and H. Shimada, On the shape of a D-brane bound state and its topology change, JHEP 03 (2009) 121 [arXiv:0901.4073] [INSPIRE].

[28] Ó.J.C. Dias, J.E. Santos and B. Way, Lumpy $A d S_{5} \times S^{5}$ black holes and black belts, JHEP 04 (2015) 060 [arXiv: 1501.06574] [InSPIRE].

[29] O. Aharony, J. Marsano, S. Minwalla, K. Papadodimas and M. Van Raamsdonk, The Hagedorn/Deconfinement Phase Transition in Weakly Coupled Large $N$ Gauge Theories, Adv. Theor. Math. Phys. 8 (2004) 603 [hep-th/0310285] [INSPIRE].

[30] T. Eguchi and H. Kawai, Reduction of Dynamical Degrees of Freedom in the Large- $N$ Gauge Theory, Phys. Rev. Lett. 48 (1982) 1063 [INSPIRE].

[31] O. Aharony, J. Marsano, S. Minwalla and T. Wiseman, Black hole-black string phase transitions in thermal $1+1$ dimensional supersymmetric Yang-Mills theory on a circle, Class. Quant. Grav. 21 (2004) 5169 [hep-th/0406210] [INSPIRE].

[32] O. Aharony, J. Marsano, S. Minwalla, K. Papadodimas, M. Van Raamsdonk and T. Wiseman, The Phase structure of low dimensional large- $N$ gauge theories on Tori, JHEP 01 (2006) 140 [hep-th/0508077] [INSPIRE].

[33] M. Hanada and T. Nishioka, Cascade of Gregory-Laflamme Transitions and U(1) Breakdown in Super Yang-Mills, JHEP 09 (2007) 012 [arXiv:0706. 0188] [INSPIRE].

[34] A.W. Peet and S.F. Ross, Microcanonical phases of string theory on $A d S_{m} \times S^{n}$, JHEP 12 (1998) 020 [hep-th/9810200] [INSPIRE]. 
[35] H. Kudoh and T. Wiseman, Connecting black holes and black strings, Phys. Rev. Lett. 94 (2005) 161102 [hep-th/0409111] [INSPIRE].

[36] O. Aharony, O. Bergman, D.L. Jafferis and J.M. Maldacena, $\mathcal{N}=6$ superconformal Chern-Simons-matter theories, M2-branes and their gravity duals, JHEP 10 (2008) 091 [arXiv:0806.1218] [INSPIRE].

[37] T. Azeyanagi, M. Fujita and M. Hanada, From the planar limit to M-theory, Phys. Rev. Lett. 110 (2013) 121601 [arXiv: 1210.3601] [INSPIRE].

[38] N. Jokela, A. Pönni and A. Vuorinen, Small black holes in global AdS spacetime, Phys. Rev. D 93 (2016) 086004 [arXiv: 1508.00859] [INSPIRE].

[39] E. Brézin and J. Zinn-Justin, Renormalization group approach to matrix models, Phys. Lett. B 288 (1992) 54 [hep-th/9206035] [INSPIRE]. 